\title{
LOW- INTENSITY PULSED ULTRASOUND APPLICATION IMPROVES BONE HEALING IN MAXILLARY SOCKETS
}

\author{
Noha M ElAdl* and Aala'a S Emara**
}

\begin{abstract}
Background: Low-Intensity Pulsed Ultrasound (LIPU) stimulates bone cells and accelerates the repair process. This article aims to evaluate its effect on bone formation by assessing bone density in a maxillary socket after extraction of a central incisor.
\end{abstract}

Methods: A RCT was designed;16 patients with badly decayed upper central incisors were selected. Eight adult patients were randomly assigned to each group. The ultrasound group were subjected to LIPU immediately after extraction through an ultrasound device and the 15 sessions scheduled at five sessions per week for three weeks. The control group patients were not exposed to any intervention. Bone density changes were measured by digital radiographs on days one and 90postoperatively.

Results: The bone density of each group was significantly increased at day 90 . The estimated mean change in bone density at day 90 was significantly larger in the ultrasound group than the control group (71.555 and 55.764 respectively) after deducting the difference at day 1.

Conclusions: the results suggest that LIPU enhances bone healing of maxillary bony extraction sockets. This may be an adjunct modality to avoid healing delays of maxillofacial bony defects as in extraction sockets and cystic defects.

KEYWORDS: Bone density, ultrasonic waves, tooth extraction, maxilla, LIPU

\section{INTRODUCTION}

Obliteration of osseous defects of the maxillofacial region is a major issue in oral surgery. Prosthetic rehabilitation and implant placement is usually hindered due to the bone loss that occurs after dental extraction ${ }^{(1)}$. Normal healing of bone undergoes remodeling through stages of bone resorption and deposition along a period of time and with periods of overlapping steps ${ }^{(2)}$. Although the resorptive phase may be quite detrimental on the width and / or height of the alveolar ride after extraction;

* Surgery and Oral Medicine Department, Oral and Dental Research Division, National Research Centre

** Oral and Maxillofacial Surgery Department, Faculty of Dentistry, Cairo University, Cairo, Egypt 
it may be beneficial for remodeling and maintaining the normal ridge configuration ${ }^{(3)}$. Local bone injury causes an inflammatory reaction which is reported to influence bone healing process ${ }^{(4)}$. Bone defects within the critical size can heal spontaneously under suitable physiological environmental conditions due to the innate regenerative ability of bone. However, this normal healing process takes a significant amount of time and new bone generation takes place slowly because of decreased blood supply and insufficient mineral content (calcium and phosphorus) to strengthen and harden the newly forming bone ${ }^{(5)}$.

Application of different healing enhancers for the bone healing has been reported. Laser application for example has been reported to enhance faster healing of bony defects ${ }^{(6)}$. The cost of laser on the other hand makes its application not feasible in many low-income countries. Moreover, Ultrasound (US) is a propagating pressure wave that delivers mechanical energy to tissues and is almost available in all hospital settings and is affordable ${ }^{(7)}$. These waves are longitudinal and consist of alternating areas of compression and rarefaction and are beyond $20,000 \mathrm{~Hz}$ (which is the upper limit of audible waves) ${ }^{(8)}$. Ultrasound is used widely in medicine as a therapeutic, operative, and diagnostic tool ${ }^{(9-12)}$. Therapeutic and operative ultrasounds use intensities as high as 1 to $3 \mathrm{~W} / \mathrm{cm}^{2}$ and can cause considerable heat generation in living tissues. To take full advantage of this energy absorption, physical therapists often use such levels of ultrasound acutely to decrease joint stiffness, to reduce pain and muscle spasms, and to improve muscle mobility ${ }^{(13)}$. The effect of ultrasound has been attributed to the increased blood flow it causes.

The purpose of this study was to evaluate the effect of Low Intensity Pulsed Ultrasound (LIPU) on bone healing in a maxillary extraction socket. We hypothesize that LIPUS will enhance bone healing and will be evident as higher bone density at the end of the followup period than the control group.

\section{MATERIAL AND METHODS}

This research was approved by Medical Research Ethical Committee and all included patients signed a printed consent of participation in this clinical trial. This randomized controlled trial complied with the principles of the Declaration of Helsinki and followed the CONSORT guidelines (Figure 1).

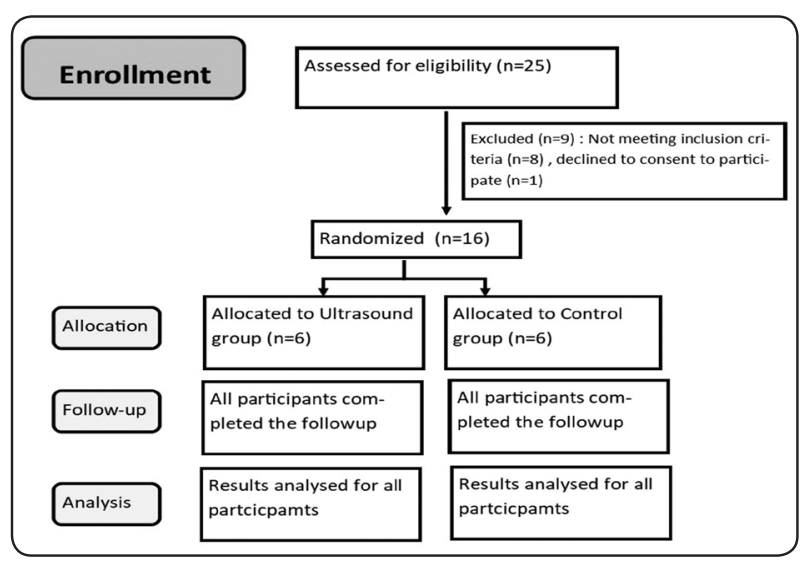

Fig. (1) Consort Flowchart showing enrollment, allocation , follow-up and analysis of the eligible subjects

\section{A. Study Design/Samples}

To address the research purpose, the investigators designed and implemented a prospective randomized control trial. The study population was composed of patients presenting for evaluation and later on management of badly decayed maxillary central incisors. After evaluation and assessment the included patients were scheduled for extraction of the maxillary central incisor. Patients with underlying medical conditions interfering with bone healing (uncontrolled diabetes/ osteoporosis) were excluded. The study was conducted during the period from February 2018 to September 2019.

\section{B. Study Variables}

After the preoperative assessment the included patients scheduled for extraction of the maxillary central incisor were divided into two groups using RANDOM.ORG. and concealed envelopes were 
used for allocation. In this study, primary predictor variables are treatment groups.

Study Group (A) (Ultrasound group): LIPU was applied after the extraction of the maxillary central incisor (Prosound ULS-1000, MedServe) ${ }^{1}$.It was applied to a single point at the extraction site. The sessions were repeated for 15 days, 5 sessions per week for a period of three weeks with the following parameters: intensity $40 \mathrm{~mW} / \mathrm{cm}^{2}$, pulse rate: $1: 1$ ultrasound signal (50\% duty cycle), frequency 1 $\mathrm{MHz}$ and the operation period 20 minutes/session (Figures 2,3).

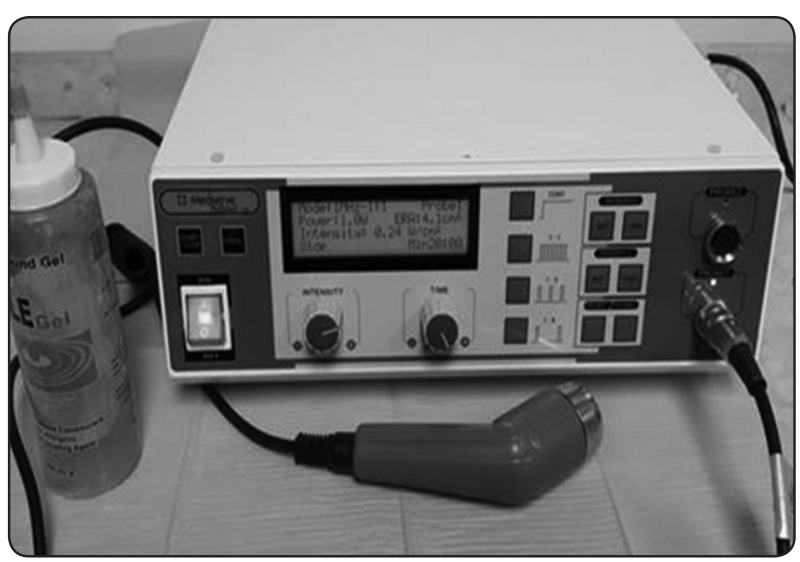

Fig. (2) Photograph showing the ultrasound device

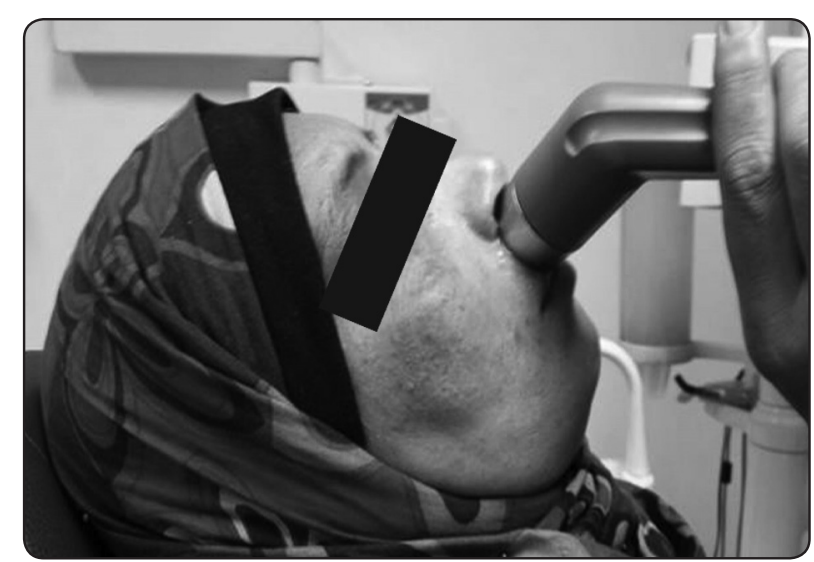

Fig. (3) Photograph showing the application of the ultrasound

Control group (B) : These patients were not subjected to any intervention and were left for the normal healing process.

The primary outcome assessed was bone healing.

\section{Data Collection Methods}

Direct measurement of bone density was done using DIGORA. The bone density changes were assessed at days 1 and 90 within and between both groups. The radiographic assessment of bone density changes was done by using size 2 photostimulable plates (PSP) of the Digora Optime imaging system (Soredex, Tuusula, Finland)i. This was repeated at days 1 and 90 postoperatively (figure 4). Digital radiography ${ }^{\text {ii }}$ operating at a tube voltage of $70 \mathrm{kVp}$ and tube current of $7 \mathrm{~mA}$ at 0.08 second was used at these time points. The bone density was measured in Pixel units.

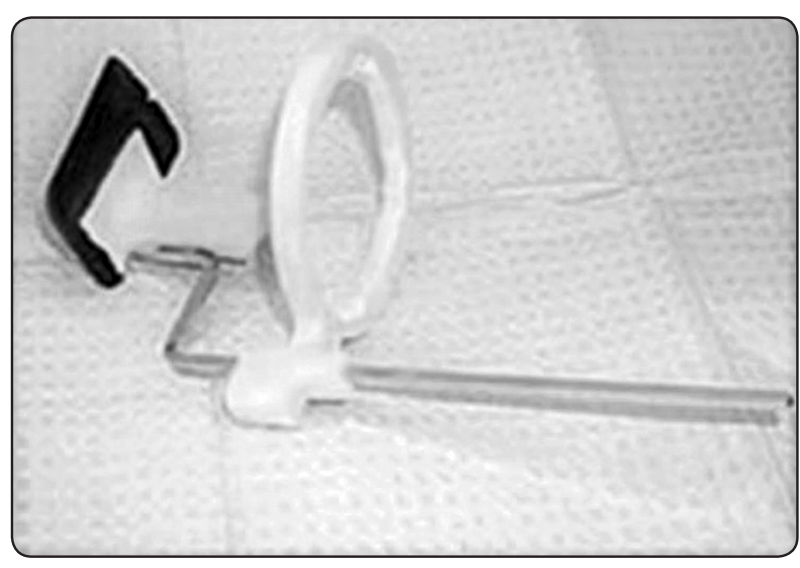

Fig. (4) Photograph showing the Rinn holder and the imaging plate of the Digora

The area density index was recorded by marking a pre-measured standard rectangular area including the area of the extraction socket to calculate the mean density of that region. Since the software does not enable hand free area selection, rectangular measurements were made with locations standardized for reproducibility by using the "start and end" as well as the " $x$ and $y$ coordinate" facilities supplied by the DIGORA software tool box. The Line Density Index was also recorded. Six horizontal lines were drawn equidistantly inside the area of the defect. The mean of the six readings was taken. The mean of the trials for each method of measurement (ten trials in total) was pooled and saved for further statistical analysis. 


\section{Data Analysis}

Data was analyzed by descriptive and bivariate statistics that were computed.

\section{RESULTS}

Sixteen healthy adult patients were included in this study with an age range of 20-44 presenting with badly decayed maxillary central incisors indicated for extraction. Participants of the US group were subjected to LIPU through an ultrasound device applied to one point at the surgical defect. Control group patients were not subjected to LIPU.

\section{A. Comparison of Bone Density within each group (at 1 and 90 day timepoints)}

In Group A (Ultrasound Group); a statistically significant difference was recorded between days 1 and day 90 (19.563) densities; with a p-value < 0.001 . The highest mean bone density value was recorded at day 90 (71.555) while the least was at day 1 (49.992). the Control group (Group B) on the other hand had a statistically insignificant difference between days 1 and 90 (2.775) where $p=0.067$. The highest bone density mean value was recorded at day 90 (55.764) and the least bone density on day 1 (52.99) (Figure 5).

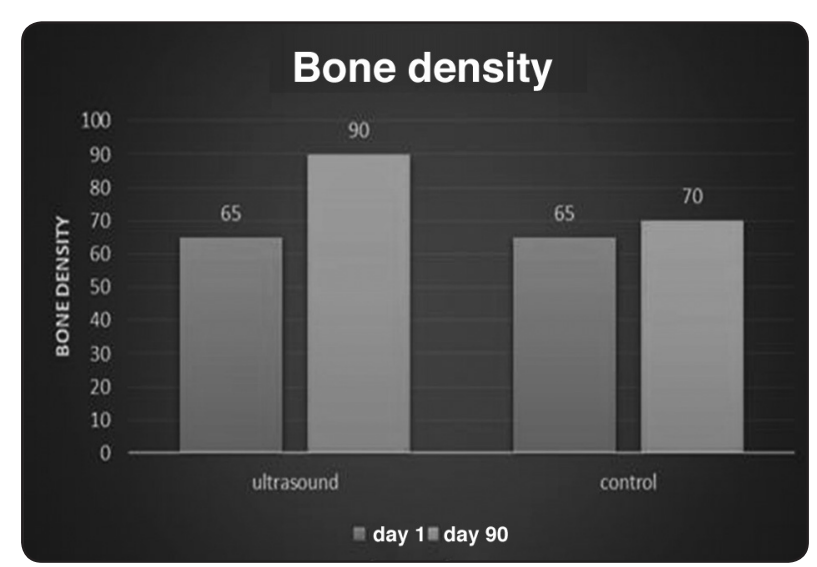

Fig. (5) Bar graph showing the bone density index at Days 1 and 90 of each group

\section{B. Comparison of Bone Density between the groups}

At day 1; there was a statistically insignificant difference between the ultrasound and control groups (49.992 and 52.991 respectively) with a p-value of 0.145 . On the other hand, at day 90 ; there was a statistically significant difference in favor of the ultrasound group with a p-value $<0.001$. (Tables 1,2).

TABLE (1) Showing the mean and SD of the pixel units in the study group

\begin{tabular}{|c|c|c|}
\hline \multirow{2}{*}{ Geriod } & \multicolumn{2}{|c|}{ Ultrasound (A) } \\
\hline Day1 & Mean & \pm SD \\
\hline Day 90 & 75.76 & $\pm 7.06^{\mathrm{a}}$ \\
\hline P-value & \multicolumn{2}{|c|}{.006* $^{*}$} \\
\hline
\end{tabular}

TABLE (2) Showing the mean and SD of the pixel units in the control group

\begin{tabular}{|c|c|c|}
\hline \multirow{2}{*}{ Group } & \multicolumn{2}{|c|}{ Control (B) } \\
\cline { 2 - 3 } Deriod & Mean & \pm SD \\
\hline Day 1 & 56.39 & $\pm 8.74^{\mathrm{a}}$ \\
\hline Day 90 & 70.04 & $\pm 6.48^{\mathrm{b}}$ \\
\hline P-value & \multicolumn{2}{|c|}{.001* $^{2}$} \\
\hline
\end{tabular}

\section{DISCUSSION}

Post-extraction bony defects are a common treatment dilemma for oral surgeons. The normal process of a healing socket starts with the organization of the formed blood clot which is then followed by bone deposition and resorption processes to allow remodeling of the area to its final form ${ }^{(11)}$. For prosthetic rehabilitation to be possible in a timely manner after extraction, the resorptive/depository phase should lead to a ridge of acceptable with and 
height to allow implant placement. To enable that; promoting bone healing has followed several approaches such as laser application for example ${ }^{(12)}$, but we decided to test the use of LIPU waves due to their availability in most hospital settings in lowincome countries and the ease and safety of application. Ultrasound waves were found to enhance bone healing due to one of several theories ${ }^{(13)}$, of which the most agreeable is that stating that the improved angiogenesis leads to improved cellular differentiation and ultimately osteogenic activity. This was in accordance with the results of our studies which showed that the images of the cases treated with the LIPU had a higher density increase after the 90 day period than the sham (non-treated group).

Applying osteogenic enhancing modalities to promote bone repair is important to preserve the morphology of the maxillary ridge. Loss of bone contour and surrounding tissue complicates further prosthetic rehabilitation (implant placement for example) as it retards regaining of function and mechanical strength and reduces the ability to prevent infection ${ }^{(14-15)}$.

Ultrasound is a form of energy that is transmitted into and through living tissues as acoustic pressure waves above the human audible range. These waves transfer mechanical energy into tissues which are propagated by ultrasound. These waves are longitudinal waves consisting of areas of compression and rarefaction ${ }^{(5)}$. Ultrasound waves with intensity $\leq 50 \mathrm{~mW} / \mathrm{cm}^{2}$ (Low Itensity Pulsed Ultrasound LIPU) were reported in many studies stimulating bone healing ${ }^{(16-20)}$. On the other hand, high intensity waves above $100 \mathrm{~mW} / \mathrm{cm}^{2}$ have been reported to delay bone healing ${ }^{(21)}$.

The LIPU was used in our study by applying it to the extraction socket for 15 sessions aiming to enhance bone healing. The exact theory behind this is unclear although the theory proposing increased angiogenesis in the healing bone is widely supported ${ }^{(22-24)}$. An in vitro study reported that human mandibular osteoblasts could be stimulated to proliferate and produce angiogenesis-related cytokines. This supports the theory that maxillofacial bone healing may be stimulated by US waves ${ }^{(25)}$. In our study LIPU was applied for 15 sessions starting from the second postoperative day. This protocol was in line with an earlier report on the timing of LIPU application and how that affects healing of mandibular fractures in which the fracture sites were exposed to LIPU for 15 days, 5 sessions per week for a period of three weeks ${ }^{(26)}$. On the other hand, another treatment protocol was proposed; which showed that continuous treatment with LIPU through a 24-day period led to higher mechanical properties compared to bone that underwent LIPU treatment for only 8 days ${ }^{(20)}$.

The bone density was measured at days 1 and 90 postoperatively in this study. This timeline was in line with a previous report in which cone beam digital radiographs were used to assess the bone density within bony cavities at similar time intervals with significant results ${ }^{(27)}$. This is supported by the natural healing process which is said to allow for deposition of woven bone which calcifies within 3-4 months. So the 90 day period we chose was sufficient to identify whether the application of LIPU had an effect on the forming bone density.

The use of radiographs for detection of bone formation in healing defects is widely accepted. It is therefore helpful in clinical situations due to the continuity of its measurements and non-invasive nature. An increase in bone radioopacity and so an image of higher optical density of healing defects is the radiographic sign of healing. Although the use of Computed Tomography (CT) is more precise; its high cost makes it difficult to be used for routine follow up exams ${ }^{(26-27)}$. The DIGORA we used assess bone density according to the opacity of the image, that is to say the assessment is based on the image density. A linear or area density is evaluated and a mean value is calculated. 
Results of our study proved that LIPU application for 15 sessions in a maxillary extraction socket did improve bone density. Further studies on the use of LIPU in larger defects and with different time periods is needed. Moreover, a deeper in-vitro evaluation of the exact theory behind the effect of the LIPU on bone healing is necessary.

\section{CONCLUSIONS}

LIPU with the parameters used in the current study has significant effect on bone healing after tooth extraction in the anterior maxillary region. Prevention of pathological fractures after large bony defects (cyst enucleation for example) may be achieved by application of LIPU. However, this needs studies with a larger sample size and larger defect sizes to prove. This will be beneficial to clinicians and researchers, as it may lead to more successful early loading of dental implants by accelerating osseointegration.

Funding : This research was partially funded by National Research Center.

Ethical approval: The Ethics Committee of National Research Center approved the steps of this research.

\section{REFERENCES}

1. Von Arx T, Buser D (2006). Horizontal ridge augmentation using autogenous block grafts and the guided bone regeneration technique with collagen membranes: a clinical study with 42 patients. Clin Oral Implants Res,17(4):359-66.

2. Yang X, Qin L, Liang W, et al. (2014). New bone formation and microstructure assessed by combination of confocal laser scanning microscopy and differential interference contrast microscopy. Calcif Tissue Int, 94(3):338-47.

3. Schmidt-Bleek K, Schell H, Schulz N, et al. (2012). Inflammatory phase of bone healing initiates the regenerative healing cascade. Cell Tissue Res, 347(3):567-73.

4. Marx RE. (2007) Bone and bone graft healing. Oral and maxillofacial surgery clinics of North America, 19(4):455-66.

5. Blumenfeld I, Srouji S, Lanir Y, Laufer D, Livne E (2002). Enhancement of bone defect healing in old rats by TGF- $\beta$ and IGF-1. Experimental gerontology, 37(4):553-65.
6. Noba C, Mello-Moura AC, Gimenez T, Tedesco TK, Moura-Netto C (2018). Laser for bone healing after oral surgery: systematic review. Lasers in medical science, 33(3):667-74.

7. Schandelmaier S, Kaushal A, Lytvyn L, Heels-Ansdell D, Siemieniuk RA, Agoritsas T, Guyatt GH, Vandvik PO, Couban R, Mollon B, Busse JW. (2017). Low intensity pulsed ultrasound for bone healing: systematic review of randomized controlled trials. British Medical Journal , 22;356:j656.

8. Baker KG, Robertson VJ, Duck FA (2001). A review of therapeutic ultrasound: biophysical effects. Physical therapy ,81(7):1351-8.

9. Ziskin MC (1987). Applications of ultrasound in medicine-comparison with other modalities. Ultrasound. Springer, Boston, MA, pp. 49-59.

10. Alazzawi MMJ, Husein A, Alam MK, et al (2018). Effect of low-level laser and low intensity pulsed ultrasound therapy on bone remodeling during orthodontic tooth movement in rats. Prog Orthod, 19(1):10.

11. John PS, Poulose CS, George B. (2008). Therapeutic ultrasound in fracture healing: The mechanism of osteoinduction. Indian J Orthop , 42(4):444-7.

12. Lemes CH, da Rosa WL, Sonego CL, Lemes BJ, Moraes RR, da Silva AF. (2019). Does laser therapy improve the wound healing process after tooth extraction? A systematic review. Wound Repair and Regeneration , 27(1):102-13.

13. Dijkman BG, Sprague S, Bhandari M (2009). Low-intensity pulsed ultrasound: Nonunions. Indian J Orthop , 43(2):141-8.

14. Miller DL, Smith NB, Bailey MR, Czarnota GJ, Hynynen K, Makin IR. (2012). Bioeffects Committee of the American Institute of Ultrasound in Medicine. Overview of therapeutic ultrasound applications and safety considerations. Journal of ultrasound in medicine , 31(4):623-34.

15. Bodner L (1998). Osseous regeneration in the jaws using demineralized allogenic bone implants. Journal of CranioMaxillofacial Surgery, 26(2):116-20.

16. Grivas KN, Vavva MG, Polyzos D, et al. (2019). Effect of ultrasound on bone fracture healing: A computational mechanobioregulatory model. J Acoust Soc Am, 145(2):1048.

17. Bowers G, Feiton F, Middleton C, Glynn D, Sharp S, Mellonig J, Corio R, Emerson J, Park S, Suzuki J, Ma S. (1991). Histologic comparison of regeneration in human intrabony defects when osteogenin is combined with demineralized freeze-dried bone allograft and with purified bovine collagen. Journal of Periodontology , 62(11):690-702 
18. Hosny M, Sharawy M. Osteoinduction in young and old rats using demineralized bone powder allografts (1985). Journal of oral and maxillofacial surgery, 43(12):925-31.

19. Shimazaki A, Inui K, Azuma Y, Nishimura N, Yamano Y (2000). Low-intensity pulsed ultrasound accelerates bone maturation in distraction osteogenesis in rabbits. The Journal of bone and joint surgery British volume, 82(7):1077-82.

20. Khadra M, Kasem N, Haanaes HR, Ellingsen JE, Lyngstadaas SP (2004). Enhancement of bone formation in rat calvarial bone defects using low-level laser therapy. Oral Surg Oral Med Oral Pathol Oral Radiol Endod, 97(6):693-700.

21. Rubin C, Bolander M, Ryaby JP, Hadjiargyrou M. (2001). The use of low-intensity ultrasound to accelerate the healing of fractures. Journal of Bone and Joint Surgery, 83(2):259.

22. Erdogan O, Esen E, Ustun Y, et al (2006). Effects of lowintensity pulsed ultrasound on healing of mandibular fractures: an experimental study in rabbits. J Oral Maxillofac Surg, 64(2):180-8.
23. Nolte PA, van der Krans A, Patka P, Janssen IM, Ryaby JP, Albers GR (2001). Low-intensity pulsed ultrasound in the treatment of nonunions. Journal of Trauma and Acute Care Surgery, 51(4):693-703.

24. Furia JP, Rompe JD, Cacchio A, Maffulli N. (2010). Shock wave therapy as a treatment of nonunions, avascular necrosis, and delayed healing of stress fractures. Foot Ankle Clin, 15(4):651- 62.

25. Doan N, Reher P, Meghji S, Harris M (1999). In vitro effects of therapeutic ultrasound on cell proliferation, protein synthesis, and cytokine production by human fibroblasts, osteoblasts, and monocytes. Journal of oral and maxillofacial surgery, 57(4):409-19.

26. Chiapasco M, Rossi A, Motta JJ, Crescentini M (2000). Spontaneous bone regeneration after enucleation of large mandibular cysts: a radiographic computed analysis of 27 consecutive cases. J Oral Maxillofac Surg , 58(9):942-9.

27. Zaky AA, El Shenawy HM, Harhsh TA, Shalash M, Awad NM. (2016). Can Low Level Laser Therapy Benefit Bone Regeneration in Localized Maxillary Cystic Defects? A Prospective Randomized Control Trial. Open Access Maced J Med Sci, 4(4):720-5. 